\title{
EXTREME INVARIANT POSITIVE OPERATORS
}

\author{
BY \\ GEORGE CONVERSE, ISAAC NAMIOKA AND R. R. PHELPS( $\left.{ }^{1}\right)$
}

0 . Introduction. Suppose that $X$ and $Y$ are sets and that $A$ and $B$ are algebras of bounded real-valued functions on $X$ and $Y$ respectively, each containing the constant functions. Let $K[A, B]$ denote the convex set of all linear $T: A \rightarrow B$ such that $T 1=1$ and $T f \geqq 0$ whenever $f \in A$ and $f \geqq 0$. It is known [10] that an operator $T$ in $K[A, B]$ is an extreme point of this set if and only if $T$ is multiplicative: $T(f g)=T f T g$ for each $f, g$ in $A$. Our intention in the present paper is to obtain analogous results for certain subsets of $K[A, B]$ (rather than for more general algebras as was done in [1]). A little more precisely, we will investigate the extreme points of the set $K[A, B]_{S}$ of all operators $T$ in $K[A, B]$ which are invariant with respect to a given semigroup $S$ (under composition) of operators from $A$ into itself. (Precise definitions are given in §1.) Roughly speaking, the extreme points turn out to be those operators which are multiplicative "modulo an averaging process." We first ( $\$ 2)$ consider the case when $B$ is the algebra of real numbers, then we consider more general $B$ in $\$ 3 . ~ \$ \$ 4$ and 5 are devoted to the case when $X$ and $Y$ are compact Hausdorff spaces and $A=C(X), B=C(Y)$.

1. Preliminaries. An algebra $A$ of functions on a nonvoid set $X$ is an algebra of real-valued bounded functions on $X$ which contains all the constant functions. The norm on $A$ will always be the supremum norm. The scalar field $R$ can be considered to be an algebra of functions on a singleton. If $A$ and $B$ are algebras of functions on sets $X$ and $Y$ respectively, $K[A, B]$ denotes the convex set of all linear maps $T: A \rightarrow B$ such that $T(1)=1$ and $T(f) \geqq 0$ whenever $f \geqq 0$. In case $B=R, K[A, B]$ will be written as $K[A]$. Clearly $K[A]$ is a subset of the dual $A^{*}$ of $A$. A semigroup $S$ [i.e. a set $S$ with an associative binary operation: $\left(s, s^{\prime}\right) \rightarrow s \cdot s^{\prime}$ ] is said to act from the right on an algebra $A$ of functions if there is a map: $A \times S \rightarrow A$ (denoted by $\left.(f, s) \rightarrow f_{s}\right)$ such that:

(i) for a fixed $s$ in $S$, the map $f \rightarrow f_{s}$ belongs to $K[A, A]$, and

(ii) $\left(f_{s}\right)_{s^{\prime}}=f_{\left(s \cdot s^{\prime}\right)}$ for all $s, s^{\prime}$ in $S$.

A linear map $T: A \rightarrow B$ is called $S$-invariant if $T\left(f_{s}\right)=T(f)$ whenever $f \in A$ and $s \in S$. The convex set of all $S$-invariant elements in $K[A, B]$ (resp. $K[A]$ ) is denoted by $K[A, B]_{S}$ (resp. $K[A]_{S}$ ). The following is an important special case.

1.1 ExAmple. Let $S$ be a semigroup and let $A=l_{\infty}(S)$. Then a right action of $S$ on $A$ can be defined by $f_{s}(t)=f(s \cdot t)$ for $s, t \in S$ and $f \in A$. The semigroup $S$ is

Received by the editors September 8, 1967.

( $\left.{ }^{1}\right)$ Work on this paper was supported in part by the National Science Foundation. 
called left-amenable if $K[A]_{S}\left(=K\left[l_{\infty}(S)\right]_{S}\right)$ is nonempty. Each element of $K[A]_{S}$ (resp. $K[A]$ ) is called a left-invariant mean (resp. mean) on $l_{\infty}(S)$. The semigroups we are mostly concerned with in the present paper are left-amenable ones. By interchanging "right" and "left", we obtain the definitions of right-amenable semigroups and right-invariant means. A semigroup which is both left and right amenable is called amenable. It is well known that a commutative semigroup is amenable.

2. Extreme invariant functionals. In this section we give several characterizations of extreme points of $K[A]_{S}$, where $S$ is a left-amenable semigroup acting from the right on an algebra $A$ of functions on a nonvoid set $X$. First we observe that $K[A]_{S}$ is not empty. This fact follows immediately from Day's fixed point theorem [3] or from the following construction: Let $m$ be a left-invariant mean on $l_{\infty}(S)$, and let $x_{0}$ be a point in $X$. For each $f \in A$, define $M(f)=m_{s}\left(f_{s}\left(x_{0}\right)\right)$ (=m applied to the function $s \rightarrow f_{s}\left(x_{0}\right)$ on $S$ ); then it is easy to check that $M \in K[A]_{s}$.

In order to state the main theorem of the section we need the notion of "averaging" in $A$. Let $\Phi$ be the convex subset of $l_{1}(S)$ consisting of all elements $\varphi$ such that $\varphi(s) \geqq 0$ for each $s$ in $S,\{s: \varphi(s)>0\}$ is finite and $\|\varphi\|_{1}=\sum\{\varphi(s): s \in S\}=1$. Each element $\varphi$ in $\Phi$ defines the $\varphi$-average $f_{\varphi}$ of a function $f$ in $A$ by $f_{\varphi}=\sum\left\{\varphi(s) f_{s}: s \in S\right\}$. Note that $\left\|f_{\varphi}\right\| \leqq\|f\|$.

2.1 Theorem. Assume that a right action of a left-amenable semigroup $S$ on an algebra $A$ of functions on a nonvoid set $X$ is given. Then $K[A]_{S}$ is nonvoid, and, for an element $L$ in $K[A]_{S}$ the following conditions are equivalent.

(a) $L$ is an extreme point of $K[A]_{S}$.

(b) For each left-invariant mean $m$ on $l_{\infty}(S)$, we have

$$
m_{s}\left[L\left(f_{s} g\right)\right]=L(f) L(g) \text { for all } f, g \in A \text {. }
$$

(c) For some mean $m$ on $l_{\infty}(S)$, we have

$$
m_{s}\left[L\left(f_{s} g\right)\right]=L(f) L(g) \text { for all } f, g \in A .
$$

(d) For each $f$ in $A$, there is a sequence $\left\{\varphi_{n}\right\}$ in $\Phi$ such that

$$
\lim _{n \rightarrow \infty} L\left(f_{\varphi_{n}} g_{\varphi_{n}}\right)=L(f) L(g) \text { for all } g \in A \text {. }
$$

(The equivalence (a) $\Leftrightarrow(b)$ is due to Lloyd [8]. However, the proof we give here is quite different from Lloyd's and is shorter. Condition (d) roughly says that $L$ is "multiplicative modulo averaging". This condition is a natural generalization of the well-known theorem which states that extreme points of $K[A]$ are multiplicative.)

Proof. (a) $\Rightarrow$ (b). First assume that $0 \leqq g \leqq 1$, and define a functional $M$ on $A$ by

$$
M(f)=m_{s}\left[L\left(f_{s} g\right)\right]-L(f) L(g) .
$$


Since $m$ is left-invariant, $M\left(f_{s}\right)=M(f)$ for $f \in A, s \in S$. Moreover it is straightforward to verify that $L \pm M \in K[A]_{s}$. Since $L$ is extreme, it follows that $M=0$, i.e., (b) holds provided $0 \leqq g \leqq 1$. Condition (b) for an arbitrary $g \in A$ follows immediately from this.

(b) $\Rightarrow$ (c) Trivial.

(c) $\Rightarrow$ (d) If we consider the embedding $l_{1}(S) \rightarrow l_{\infty}(S)^{*}$, then the image of $\Phi$ is weak* dense in the set of means in $l_{\infty}(S)^{*}\left(=K\left[l_{\infty}(S)\right]\right)$. Hence there is a net $\left\{\varphi_{\alpha}\right\}$ in $\Phi$ such that $m(h)=\lim _{\alpha} \sum\left\{\varphi_{\alpha}(s) h(s): s \in S\right\}$ for each $h \in l_{\infty}(S)$. Thus, condition (c) implies that, for $f, g \in A$,

$$
L(f) L(g)=\lim _{\alpha} \sum\left\{\varphi_{\alpha}(s) L\left(f_{s} g\right): s \in S\right\}=\lim _{\alpha} L\left(f_{\varphi_{\alpha}} g\right) .
$$

Now we want to do the following: For a fixed $f$, replace the net in the right hand side by a suitable sequence in $\Phi$ so that the convergence is uniform over any bounded set of functions $g$. To this end we construct an auxiliary Hilbert space: Define a bilinear form ( , ) on $A \times A$ by $(u, v)=L(u v)$, and let

$$
N=\left\{u: u \in A, L\left(u^{2}\right)=0\right\} \text {. }
$$

Using the Schwarz inequality $L(u v)^{2} \leqq L\left(u^{2}\right) L\left(v^{2}\right)$, it is easy to verify that $N$ is an ideal in $A$. Let $P: A \rightarrow A / N$ be the quotient map and introduce a bilinear form $\langle, \quad>$ in $A / N \times A / N$ by

$$
\langle P u, P v\rangle=(u, v)=L(u v) .
$$

Then $A / N$ is an inner product space, and we shall denote by \|\| the norm resulting from $\langle$,$\rangle , i.e., \|P u\|^{2}=\langle P u, P u\rangle=L\left(u^{2}\right)$. Note that $\|P u\| \leqq\|u\|$. Let $H$ denote the real Hilbert space obtained by completing the inner product space $A / N$. We shall denote by $\langle,>$ and \|\| the extended inner product and norm on $H$ as well. Now write $z=P(L(f) \cdot 1)$; then $\left(^{*}\right)$ can be written as $\langle z, P(g)\rangle=\lim _{\alpha}\left\langle P\left(f_{\varphi_{\alpha}}\right), P(g)\right\rangle$ for all $g \in A$. Since $\left\|P\left(f_{\varphi_{\alpha}}\right)\right\| \leqq\left\|f_{\varphi_{\alpha}}\right\| \leqq\|f\|$ and $P[A]$ is dense (relative to \|\|$)$ in $H$, we see that the net $\left\{P\left(f_{\varphi_{\alpha}}\right)\right\}$ converges weakly to $z$ in $H$, i.e., $z$ belongs to the weak closure of the convex set $\left\{P\left(f_{\varphi}\right): \varphi \in \Phi\right\}$, hence $z$ belongs to the norm closure of the same set. Therefore there is a sequence $\left\{\varphi_{n}\right\}$ in $\Phi$ such that $\lim _{n}\left\|z-P\left(f_{\varphi_{n}}\right)\right\|=0$. Since, for each $g \in A$,

$$
\left|L(f) L(g)-L\left(f_{\varphi_{n}} g\right)\right|=\left|\left\langle z-P\left(f_{\varphi_{n}}\right), P(g)\right\rangle\right| \leqq\left\|z-P\left(f_{\varphi_{n}}\right)\right\| \cdot\|g\|,
$$

we have $\lim _{n} L\left(f_{\varphi_{n}} g\right)=L(f) L(g)$ uniformly over any norm bounded set of functions $g$ in $A$. Because $\left\|g_{\varphi_{n}}\right\| \leqq\|g\|$, assertion (d) is now evident.

(d) $\Rightarrow$ (a). Assume that $L$ satisfies (d) and $2 L=L_{1}+L_{2}$ for $L_{1}, L_{2} \in K[A]_{S}$. We must show that $L_{1}=L$. Suppose $f$ is an element in $A$ such that $L(f)=0$. Then, by (d), there is a sequence $\left\{\varphi_{n}\right\}$ in $\Phi$ such that $\lim _{n}\left(L\left(f_{\varphi_{n}}\right)^{2}\right)=0$. Hence, by using the Schwarz inequality, we see that

$$
0 \leqq\left[L_{1}(f)\right]^{2}=\left[L_{1}\left(f_{\varphi_{n}}\right)\right]^{2} \leqq L_{1}(1) L_{1}\left[\left(f_{\varphi_{n}}\right)^{2}\right] \leqq 2 L\left[\left(f_{\varphi_{n}}\right)^{2}\right]
$$


for each $n$. Therefore $L_{1}(f)=0$ whenever $L(f)=0$. This implies that $L_{1}=k L$ for some constant $k$. But $1=L_{1}(1)=k L(1)=k$, and hence $L_{1}=L$.

It is worth noting that, in the proofs (c) $\Rightarrow$ (d) and (d) $\Rightarrow$ (a), we did not use the fact that $S$ is left amenable. Consequently the implications (c) $\Rightarrow$ (d) $\Rightarrow$ (a) are valid for an arbitrary semigroup $S$ acting on $A$ from the right and for $L$ in $K[A]_{S}$.

3. Extreme invariant operators. We now turn to the case of the set $K[A, B]_{S}$, where $A$ and $B$ are algebras of functions on nonvoid sets $X$ and $Y$ respectively and $S$ is a left-amenable semigroup acting on $A$ from the right. Note that, under these assumptions, $K[A, B]_{S}$ is nonvoid. For, take $L \in K[A]_{S}$, and define $T: A \rightarrow B$ by $T(f)=L(f) \cdot 1$. Then it is clear that $T \in K[A, B]_{s}$. Given an operator $T \in K[A, B]_{S}$ and a point $y \in Y$, we can define an element $T^{\sim}(y)$ in $K[A]_{S}$ by $T^{\sim}(y)(f)=T(f)(y)$ for $f \in A$. It can be readily verified that if $T^{\sim}(y)$ is an extreme point in $K[A]_{S}$ for each $y$ in $Y$, then $T$ is extreme in $K[A, B]_{S}$. The converse of this assertion is false (see Theorem 4.4), and this fact presents the major difficulty in the determination of extreme points of $K[A, B]_{S}$ in general. The following assertion is useful in establishing the converse in some special cases.

3.1 Proposition. Let $A, B$ and $S$ be as above, and let $T$ be an extreme point in $K[A, B]_{S}$. Suppose that there is a left-invariant mean $m$ on $l_{\infty}(S)$ such that the function

$$
y \rightarrow m_{s}\left[T\left(f_{s} g\right)(y)\right]
$$

is in $B$ whenever $f, g \in A$. Then $T^{\sim}(y)$ is an extreme point in $K[A]_{S}$ for each $y$ in $Y$.

Proof. Choose $g$ in $A$ such that $0 \leqq g \leqq 1$, and define an operator $U: A \rightarrow B$ by

$$
U(f)(y)=m_{s}\left[T\left(f_{s} g\right)(y)\right]-(T f)(y)(T g)(y) \text { for } f \in A, y \in Y \text {. }
$$

Then the left-invariance of $m$ and the $S$-invariance of $T$ imply that $U\left(f_{s}\right)=U(f)$ for $f$ in $A$ and $s$ in $S$. It is also easy to check the remaining inequalities to show that $T \pm U \in K[A, B]_{s}$. Since $T$ is extreme, $U=0$, or $m_{s}\left[T\left(f_{s} g\right)(y)\right]=(T f)(y)(T g)(y)$ for all $y$ provided $0 \leqq g \leqq 1$. It follows immediately that, for each $y$ in $Y, T^{\sim}(y)$ satisfies condition (c) of Theorem 2.1; hence, $T^{\sim}(y)$ is extreme in $K[A]_{s}$.

Note that the converse of 3.1 is also true: If $T$ is an element in $K[A, B]_{S}$ such that $T^{\sim}(y)$ is extreme in $K[A]_{S}$ for each $y$, then the function defined by (1) is always in $B$, since, by Theorem 2.1 , the function defined by (1) is just $(T f)(T g)$.

3.2 Corollary. Let $A, B$ and $S$ be as above. If any one of the following conditions is satisfied, then an element $T$ in $K[A, B]_{S}$ is extreme in $K[A, B]_{S}$ if and only if $T^{\sim}(y)$ is an extreme point in $K[A]_{S}$ for each $y$ in $Y$.

(i) $B=l_{\infty}(Y)$,

(ii) $S$ is finite,

(iii) $S$ contains a left ideal which is a finite group. 
Proof. It is clear that under hypothesis (i) or (ii), the function (1) of Proposition 3.1 is always in $B$. Assume, now, that (iii) holds. Let $G$ be a finite group in $S$ which is a left ideal. As shown in an addendum to [4], this implies that $s G=G$ for each $s$ in $S$; indeed, if $e$ is the identity for $G$, then $s G=s(e G)=(s e) G=G$, since $s e \in G$. It follows from this remark that the following mean $m$ is invariant on $l_{\infty}(S)$ :

$$
m(u)=(1 / N) \sum\{u(s): s \in G\},
$$

where $N$ is the order of $G$. It is clear that the condition of Proposition 3.1 is satisfied for this mean.

In the above corollary, (ii) is a special case of (iii), since for a finite left-amenable semigroup $S$, condition (iii) is always satisfied. There is an extensive discussion by Granirer in [4] of semigroups which satisfy (iii).

Suppose that the action of $S$ on the algebra of functions $A$ is given by $(f, s) \rightarrow f \circ s$, where $S$ is given by a semigroup of maps of $X$ into itself. For a special class of semigroups, Granirer [7] has shown that the extreme points of $K[A, B]_{s}$ are always multiplicative. These are the extremely left amenable semigroups, which have been studied by Granirer [5], [6], [7] and which he has characterized by the following property:

If $s_{1}, s_{2} \in S$, there exists $t$ in $S$ such that $s_{1} t=s_{2} t=t$.

In particular, given $s$ in $S$, there is a $t$ in $S$ such that $s t=t$. Thus, if $L \in K[A]_{S}$, then for any $f, g$ in $A, L\left[\left(f_{s} g\right)=L\left[\left(f_{s} g\right)_{t}\right]=L\left(f_{s t} g_{t}\right)=L(f g)\right.$. For any mean $m$ on $S$, then, $m_{s}\left[L\left(f_{s} g\right)\right]=L(f g)$, so that Granirer's result follows immediately from Theorem 2.1 and Proposition 3.1.

4. Algebras of continuous functions. In this section we consider algebras of continuous functions. Let $X$ and $Y$ be compact Hausdorff spaces and let $C(X)$ and $C(Y)$ be the algebras of all continuous real valued functions on $X$ and $Y$ respectively. Let $S$ be a semigroup (not necessarily left-amenable) acting on $C(X)$ from the right. As before, we are interested in characterizing extreme points of $K[C(X), C(Y)]_{S}$; however, we have only partial answers. Recall that, for an element $T$ in $K[C(X), C(Y)]_{S}$, we can define a map $T^{\sim}: Y \rightarrow K[C(X)]_{S}$ by

$$
T^{\sim}(y)(f)=T(f)(y) .
$$

Since $T^{\sim}$ is the composition of the evaluation map: $Y \rightarrow C(Y)^{*}$ and $T^{*}: C(Y)^{*} \rightarrow$ $C(X)^{*}, T^{\sim}$ is a continuous map from $Y$ into $\left(K[C(X)]_{S}\right.$, weak*). Conversely, to each continuous map $T^{\sim}: Y \rightarrow\left(K[C(X)]_{S}\right.$, weak*), equation (2) defines a unique element $T$ in $K[C(X), C(Y)]_{S}$. We now summarize the above discussion:

4.1 Lemma. Let $X, Y$ and $S$ be as above. Then the correspondence $T \rightarrow T^{\sim}$ gives a one-to-one, affine, onto map: $K[C(X), C(Y)]_{S} \rightarrow \operatorname{Map}\left(Y, K[C(X)]_{S}\right)$, where Map $\left(Y, K[C(X)]_{S}\right)$ is the space of all continuous maps $Y \rightarrow\left(K[C(X)]_{s}\right.$, weak*) with the obvious affine structure. 
We may also generalize the remark made in the beginning of $\S 3$. The proof is immediate.

4.2 Proposition. Let $X, Y$ and $S$ be as above, and let $T$ be an element in $K[C(X), C(Y)]_{S}$ such that $T^{\sim}(y)$ is extreme in $K[C(X)]_{s}$ for each y belonging to a dense subset of $Y$. Then $T$ is extreme in $K[C(X), C(Y)]_{S}$.

In particular, if $T$ is an element in $K[C(X), C(Y)]_{s}$ such that $T^{\sim}(y)$ is extreme in $K[C(X)]_{S}$ for all $y$ in $Y$, then $T$ is extreme. That the converse of this statement is false follows from Theorem 4.4 (and the subsequent remark), where we characterize those actions for which the converse is true.

The following lemma is fairly well known; the proof is due to Choquet.

4.3 LEMMA. Let $X$ be a compact Hausdorff space and let $S$ be a semigroup acting on $C(X)$ from the right. Then $K[C(X)]_{S}$ is a simplex in the sense of Choquet [11].

Proof. Let $C(X)_{S}^{*}$ be the subspace of all $S$-invariant elements of $C(X)^{*}$. Now $C(X)^{*}$ is a vector lattice, and if we show that $C(X)_{S}^{*}$ is closed under these lattice operations, then $C(X)_{S}$ is a lattice under the partial order induced by the cone generated by $K[C(X)]_{s}$. By the usual translation argument, it is sufficient to prove that $\lambda^{+}$is $S$-invariant whenever $\lambda$ is. For $s \in S$ and $\mu \in C(X)^{*}$, define $s \cdot \mu \in C(X)^{*}$ by $s \cdot \mu(f)=\mu\left(f_{s}\right), f \in C(X)$. Then $\mu$ is $S$-invariant if and only if $s \cdot \mu=\mu$ for each $s \in S$. Now let $\lambda$ be $S$-invariant and $s \in S$. Then $\lambda^{+} \geqq 0$ and $\lambda^{+} \geqq \lambda$ imply $s \cdot\left(\lambda^{+}\right) \geqq 0$ and $s \cdot\left(\lambda^{+}\right) \geqq s \cdot \lambda=\lambda$. Hence $s \cdot\left(\lambda^{+}\right) \geqq \lambda^{+}$, and it follows that $\left\|s \cdot\left(\lambda^{+}\right)-\lambda^{+}\right\|$ $=\left\langle s \cdot\left(\lambda^{+}\right)-\lambda^{+}, 1\right\rangle=\left\langle s \cdot\left(\lambda^{+}\right), 1\right\rangle-\left\langle\lambda^{+}, 1\right\rangle=0$. Hence $s \cdot\left(\lambda^{+}\right)=\lambda^{+}$for each $s \in S$, or $\lambda^{+}$is $S$-invariant.

4.4 TheOREM. Let $X$ be a compact Hausdorff space and let $S$ be a semigroup (not necessarily left-amenable) acting on $C(X)$ from the right. Then the following two statements are equivalent.

(a) The set of all extreme points of $K[C(X)]_{S}$ is weak* compact.

(b) For an arbitrary compact Hausdorff space $Y$, an element $T$ in $K[C(X), C(Y)]_{S}$ is extreme if and only if $T^{\sim}(y)$ is extreme in $K[C(X)]_{S}$ for each $y$ in $Y$.

Proof. (a) $\Rightarrow(\mathrm{b})$. Let $E$ be the set of all extreme points of $K[C(X)]_{S}$, with the weak* topology. By hypothesis, $E$ is a compact Hausdorff space, and we can define $\phi: C(X) \rightarrow C(E)$ by $\phi(f)(e)=\langle e, f\rangle$ for all $e$ in $E$. The "resultant" map $r: K[C(E)] \rightarrow K[C(X)]_{s}$ can be expressed as: $r(M)(f)=\langle M, \phi(f)\rangle$, for $M \in K[C(E)]$ and $f \in C(X)$. Since $E$ is compact and $K[C(X)]_{S}$ is a simplex (Lemma 4.3), we know that (cf. [11]) $r$ is a homeomorphism of $\left(K[C(E)]\right.$, weak*) and $\left(K[C(X)]_{S}\right.$, weak*). Hence using Lemma 4.1 twice, we obtain the following identifications:

$$
\begin{aligned}
K[C(X), C(Y)]_{S} & =\operatorname{Map}\left(Y, K[C(X)]_{S}\right)=\operatorname{Map}(Y, K[C(E)]) \\
& =K[C(E), C(Y)] .
\end{aligned}
$$


Using Corollary 3.2 for the trivial semigroup acting trivially on $C(E)$, we see that $T \in K[C(X), C(Y)]_{S}$ is extreme if and only if $r^{-1} T^{\sim}(y)$ is extreme in $K[C(E)]$ for each $y \in Y$. But extreme points of $K[C(E)]$ are simply evaluations at points of $E$, so that the image under $r$ of the set of all extreme points of $K[C(E)]$ is precisely the set $E$. It follows that $T$ is extreme if and only if $T^{\sim}(y) \in E$ for each $y$ in $Y$.

(b) $\Rightarrow$ (a). Let $E$ be the set of all extreme points of $K[C(X)]_{S}$ and let $Y$ be the weak* closure of $E$. Then $Y$ is compact and Hausdorff relative to the weak* topology. Let $T^{\sim}$ be the inclusion map $Y \rightarrow K[C(X)]_{S}$. Then by Lemma 4.1, $T^{\sim}$ defines an element $T$ in $K[C(X), C(Y)]_{s}$, and $T$ is extreme there by Proposition 4.2. Then by (b), $T^{\sim}(y)=y \in E$ for all $y$ in $Y$, i.e. $Y \subset E$. Hence $E$ is weak* compact.

An example (due to Choquet) which shows that the extreme points of $K[C(X)]_{S}$ need not be weak* compact is described in [11, p. 83].

In the next theorem we give two conditions for the set of extreme points of $K[C(X)]_{S}$ to be weak* compact.

4.5 THEOREM. Let $X$ be a compact Hausdorff space, and let $S$ be a left-amenable semigroup acting on $C(X)$ from the right. If one of the following conditions is satisfied, then the set of all extreme points of $K[C(X)]_{S}$ is weak* compact.

(i) The action of $S$ is weakly almost periodic, i.e., for each $f$ in $C(X)$, the weak closure of the set $S(f)=\left\{f_{s}: s \in S\right\}$ in $C(X)$ is weakly compact.

(ii) $S$ contains a left ideal which is a finite group (cf. remark following Corollary 3.2).

Proof. (i) Let $m$ be a left invariant mean on $l_{\infty}(S)$; then there is a net $\left\{\varphi_{\alpha}\right\}$ in $\Phi$ such that $\varphi_{\alpha} \rightarrow m$ relative to the weak* topology, where $\Phi$ is regarded as being embedded in $l_{\infty}(S)^{*}$. By Theorem 2.1, an element $\lambda$ in $K[C(X)]_{S}$ is extreme there if and only if $m_{s}\left[\lambda\left(f_{s} g\right)\right]=\lim _{\alpha} \lambda\left(f_{\omega_{\alpha}} g\right)=\lambda(f) \lambda(g)$ for all $f$ and $g$. In order to prove that the set of extreme points of $K[C(X)]_{S}$ is weak* compact, it is sufficient to prove that, for fixed $f$ and $g$, the set $\Lambda_{f, g}=\left\{\lambda: m_{s}\left[\lambda\left(f_{s} g\right)\right]=\lambda(f) \lambda(g)\right\}$ is weak* closed in $C(X)^{*}$. Since the closure of the convex hull $\left\{f_{\varphi}: \varphi \in \Phi\right\}$ of $S(f)$ is weakly compact we may assume that weak- $\lim _{\alpha} f_{\varphi_{\alpha}}=f_{0}$. Then weak- $\lim _{\alpha} f_{\varphi_{\alpha}} g=f_{0} g$, and consequently $m_{s}\left[\lambda\left(f_{s} g\right)\right]=\lambda\left(f_{0} g\right)$. It follows that $\Lambda_{f, g}=\left\{\lambda: \lambda\left(f_{0} g\right)=\lambda(f) \lambda(g)\right\}$, which is obviously weak* closed.

(ii) As noted in the proof of Corollary 3.2, in this case there is a left-invariant mean $\varphi$ on $l_{\infty}(S)$ which belongs to $\Phi$. In view of Theorem 2.1, an element $\lambda$ in $K[C(X)]_{S}$ is extreme there if and only if $\lambda\left(f_{\varphi} g\right)=\lambda(f) \lambda(g)$ for all $f, g \in C(X)$. Hence the set of all extreme points of $K[C(X)]_{S}$ is weak* closed.

4.6 Corollary. Let $X$ and $Y$ be compact Hausdorff spaces, and let $S$ be a leftamenable semigroup acting from the right on $C(X)$ satisfying (i) or (ii) of Theorem 4.5. Then for an element $T$ in $K[C(X), C(Y)]$, the following statements are equivalent.

(a) $T$ is extreme in $K[C(X), C(Y)]_{S}$, 
(b) $T^{\sim}(y)$ is extreme in $K[C(X)]_{s}$ for each $y$ in $Y$,

(c) For some (or every) left-intariant mean $m$ on $l_{x}(S)$, we have

$$
m_{s}\left[T\left(f_{s} g\right)(y)\right]=T(f)(y) T(g)(y)
$$

for all $f, g \in C(X)$ and $y \in Y$.

Proof. Immediate from Theorem 4.5, Theorem 4.4 and Theorem 2.1.

4.7 Examples. We list some instances in which the right action of $S$ on $C(X)$ is weakly almost periodic.

(i) The semigroup $S$ is finite.

(ii) The semigroup $S$ is compact and, for each $f$ in $C(X)$, the map $s \rightarrow f_{s}$ is weakly continuous. This will be the case if $S$ is compact metrizable and, for each $f \in C(X)$ and $x \in X$, the map $s \rightarrow f_{s}(x)$ is continuous. (Use Lebesgue's bounded convergence theorem.)

(iii) The space $X$ is compact metric and $f_{s}=f \circ s$ for $s$ in a semigroup $S$ of contractions on $X$ (i.e. $d\left(s(x), s\left(x^{\prime}\right)\right) \leqq d\left(x, x^{\prime}\right)$ for all $x, x^{\prime} \in X$ and $s \in S$ ). In this case the set $\left\{f_{s}: s \in S\right\}$ is equicontinuous, and hence it is relatively compact in $C(X)$ with respect to the norm topology.

5. Amenable semigroups acting on $C(X)$. Several of the characterizations of extreme invariant measures and operators presented above take on particularly simple forms in case the semigroup $S$ admits both a right and a left invariant mean, i.e., when $S$ is amenable. We require a preliminary lemma.

5.1 Lemma. Suppose that $X$ is a compact Hausdorff space, that $S$ is amenable and that the action of $S$ from the right on $C(X)$ is weakly almost periodic. Then there exists a linear projection $P$ from $C(X)$ onto the closed subspace

$$
D=\left\{f \in C(X): f_{s}=\text { ffor each } s \text { in } S\right\}
$$

which satisfies each of the following:

(i) $P \geqq 0, P 1=1$ and $P(f)=P\left(f_{s}\right)$ for $f$ in $C(X)$, $s$ in $S$.

(ii) The operator $P$ is given explicitly by $(P f)(x)=m_{s}\left[f_{s}(x)\right]$, where $f \in C(X)$, $x \in X$ and $m$ is some left-intariant mean on $S$.

(iii) For any topological vector space $E$ and any continuous linear $T: C(X) \rightarrow E$, we have $T P=T$ if and only if $T\left(f_{s}\right)=T f$ for each $f$ in $C(X), s$ in $S$.

Proof. Suppose that $f \in C(X)$; since the closure of the convex hull of $S(f)$ is weakly compact, and since $S$ acts on the right and is right amenable, the fixedpoint theorem of Day[3] shows that there exists at least one $g$ in the closed convex hull of $S(f)$ such that $g=g_{s}$ for each $s$ in $S$. Choose a left-invariant mean $m$ on $S$ and suppose that $\varepsilon>0$. We can choose $s_{1}, \ldots, s_{n}$ in $S$ and $\lambda_{1}, \ldots, \lambda_{n}$ with $\lambda_{i}>0$, $\sum \lambda_{i}=1$, such that

$$
\left\|g-\sum \lambda_{i} f_{s_{i}}\right\| \leqq \varepsilon
$$


Thus, if $x \in X$, then for each $s$ in $S$ we have

$$
\left|g(x)-\sum \lambda_{i} f_{s_{i} s}(x)\right| \leqq \varepsilon
$$

If we apply $m$ to this (bounded) function of $s$, we obtain

$$
\varepsilon \geqq\left|g(x)-\sum \lambda_{\imath} m_{s}\left[f_{s_{s}}(x)\right]\right|=\left|g(x)-m_{s}\left[f_{s}(x)\right]\right|,
$$

which shows that $g(x)=m_{s}\left[f_{s}(x)\right]$ for each $x$. Defining $(P f)(x)=m_{s}\left[f_{s}(x)\right]$, we see that $P f=g \in D$. It is clear that $P$ is linear, satisfies (i) and (ii), that $P^{2}=P$ and that the range of $P$ is $D$. To prove (iii), suppose that $T\left(f_{s}\right)=T f$ for each $f$ and $s$. Then $T[S(f)]=\{T f\}$ for each $f$, and by continuity and linearity, the same is true of the closed convex hull of $S(f)$. But $g=P f$ is contained in the latter, so $T(P f)=T f$. Conversely, if $T P=T$, then $T\left(f_{s}\right)=(T P)\left(f_{s}\right)=T(P f)=T f$ for each $f$ and $s$.

Note that we do not assert that the subspace $D$ above is a subalgebra, although this will certainly be the case if each $s$ in $S$ is multiplicative.

5.2 COROLlary. Under the above hypothesis on $X$ and $S$, the following assertions concerning a measure $\mu$ in $K[C(X)]_{S}$ are equivalent:

(i) The measure $\mu$ is extreme in $K[C(X)]_{s}$.

(ii) For any $f, g$ in $C(X), \mu(f \cdot P g)=\mu(f) \mu(g)$.

(iii) For any $f, g$ in $C(X), \mu(P f \cdot P g)=\mu(f) \mu(g)$.

(iv) For any $f, g$ in $D, \mu(f g)=\mu(f) \mu(g)$.

Proof. This corollary is merely a reformulation of Theorem 2.1 , taking into account assertion (iii) of the previous theorem and the fact that $\{P\}$ is a oneelement semigroup. In particular, the set $\Phi$ of finite means contains only one element, which yields (iii) from 2.1 (d).

5.3 THEOREM. Suppose that $X$ and $Y$ are compact Hausdorff spaces and that $S$ is an amenable semigroup which acts weakly almost periodically on the right on $C(X)$. Let $P$ be the projection defined in Lemma 5.1, and let $D$ be its range. If $T \in K[C(X), C(Y)]_{S}$, then the following assertions are equivalent:

(a) $T$ is extreme in $K[C(X), C(Y)]_{s}$.

(b) $T^{\sim}(y)$ is extreme in $K[C(X)]_{s}$ for each $y$ in $Y$.

(c) $T(f \cdot P g)=T f T g$ for each $f, g$ in $C(X)$.

(d) $T(f g)=T f T g$ for each $f, g$ in $D$.

Proof. This is immediate from Corollary 4.6 and Corollary 5.2.

6. An example. In connection with Theorem 4.5 it is worth noting that the extreme points of $K[C(X)]_{S}$ can be closed, even though the action of $S$ is not weakly almost periodic. This is shown by the following simple example, where there are only two extreme points.

ExAmPLE. Let $X=[0,1]$ and let $S$ be the positive integers, acting on the right on $C[0,1]$ by

$$
f_{n}(x)=f\left(x^{2^{n}}\right), \quad f \in C[0,1], 0 \leqq x \leqq 1
$$


Then there are exactly two extreme points in $K[C(0,1)]_{S}$, the evaluation functionals $f \rightarrow f(0)$ and $f \rightarrow f(1)$. Furthermore, $S$ is not weakly almost periodic.

Proof. It is clear that the above definition does give a semigroup action; it is just composition of $f$ with the semigroup of iterates (under composition) of the function $x \rightarrow x^{2}$. Suppose that $\mu \in K[C(X)]_{s}$ and that $k$ is a positive integer. If $f(x)=x^{k}$, then $f_{n}(x)=x^{k 2^{n}} \rightarrow \delta_{1}(x)$, where $\delta_{1}(x)=0,0 \leqq x<1, \delta_{1}(1)=1$. By the Lebesgue bounded convergence theorem, $\mu(f)=\mu\left(f_{n}\right) \rightarrow \mu\left(\delta_{1}\right)$, so $\mu(f)=\mu\left(\delta_{1}\right)$. Thus, if $p(x)=\sum_{k=0}^{n} a_{k} x^{k}$, we have

$$
\begin{aligned}
\mu(p) & =a_{0}+\left(\sum_{k=1}^{n} a_{k}\right) \mu\left(\delta_{1}\right) \\
& \left.=p(0)+\left[p^{(} 1\right)-p(0)\right] \mu\left(\delta_{1}\right) \\
& =\mu\left(\delta_{1}\right) p(1)+\left[1-\mu\left(\delta_{1}\right)\right] p(0) .
\end{aligned}
$$

Since this is true for the dense set of all polynomials, it holds for every $f$ in $C[0,1]$. Since $0 \leqq \mu\left(\delta_{1}\right) \leqq 1$, we have shown that each invariant probability measure $\mu$ is a convex combination of the two point masses at 0 and 1 . The latter are clearly extreme (and invariant) and hence they are the only extreme points. To see that the action of $S$ is rot weakly almost periodic, consider the set of iterates $\left\{g_{n}\right\}$ of $g(x)=x$; they converge pointwise to $\delta_{1}$. If the weak closure of $\left\{g_{n}\right\}$ were weakly compact, there would be a subset of $\left\{g_{n}\right\}$ converging weakly-hence pointwiseto a continuous function. The only choice for such a limit function is $\delta_{1}$, which is not continuous.

It is interesting to note (and easy to prove) that if ext $K[C(X)]_{S}$ consists of finitely many measures $\mu_{1}, \ldots, \mu_{n}$, then (for any compact $Y$ ), every operator $T$ in $K[C(X), C(Y)]_{s}$ has the form

$$
(T f)(y)=\sum_{i=1}^{n} a_{i}(y) \mu_{i}(f), \quad y \in Y, f \in C(X)
$$

where each $a_{i} \in C(Y), a_{i} \geqq 0$ and $\sum a_{i}=1$. (The continuity of the $a_{i}$ follows from the fact that $K[C(X)]_{s}$ is the simplex with vertices $\mu_{1}, \ldots, \mu_{n}$, together with the continuity of $y \rightarrow T^{\sim} y$.) Such an operator is extreme if and only if $a_{i}^{2}=a_{i}$ for each $i$, since this is precisely what is needed in order that $T^{\sim} y$ be one of the $\mu_{i}$, for each $y$.

There is a well-known characterization of extreme invariant measures as ergodic measures (see e.g. [11]), and it is of interest to compare this with the characterizations given in Theorem 2.1. Let $S$ be a semigroup of measurable transformations on the measure space $T$; a probability measure $\mu$ on $T$ is said to be ergodic if $\mu(G)=0$ or 1 whenever $G$ is a measurable subset of $T$ such that $\mu\left(G \triangle s^{-1} G\right)=0$ for each $s$ in $S$. If we let $A$ be the algebra generated by the characteristic functions of measurable subsets of $T$, and let $L$ be the linear functional on $A$ defined by $L(f)=\mu(f)(f \in A)$, then this may be reformulated as $L$ is extreme in $K[A]_{S}$ if and only if $L\left(f^{2}\right)=(L f)^{2}$ whenever $f \in A, f^{2}=f$ and $L\left[\left(f-f_{s}\right)^{2}\right]=0$ for each $\sin S$. Obviously 
this must be equivalent (for this particular $A$ and left-amenable $S$ ) to the characterization given in Theorem 2.1, but we see no simple way to prove this equivalence directly. (Note that the above characterization does not require that $S$ be leftamenable.)

\section{BIBLIOGRAPHY}

1. F. F. Bonsall, Joram Lindenstrauss and R. R. Phelps, Extreme positive operators on algebras of functions, Math. Scand. 18 (1966), 161-182.

2. George Converse, Extreme positive operators on $C(X)$ which commute with certain operators, Trans. Amer. Math. Soc. (to appear).

3. M. M. Day, Fixed-point theorems for compact convex sets, Illinois J. Math. 5 (1961), 585-590.

4. E. Granirer, On amenable semigroups with a finite dimensional set of invariant means. I, II, Illinois J. Math. 7 (1963), 32-48, 49-58.

5. —-, Extremely amenable semigroups, Math. Scand. 17 (1965), 177-197.

6. —_, Extremely amenable semigroups. II, Math. Scand. 20 (1967), 93-113.

7. - Extremely amenable semigroups. III, Math. Scand. (to appear).

8. S. P. Lloyd, A mixing condition for extreme left invariant means, Trans. Amer. Math. Soc. 125 (1966), 461-481.

9. I. Namioka, On certain actions of semigroups on L-spaces, Studia Math. 29 (1967), 63-77.

10. R. R. Phelps, Extreme positive operators and homomorphisms, Trans. Amer. Math. Soc. 108 (1963), 265-274.

11. —- Lectures on Choquet's Theorem, Van Nostrand, Princeton, N. J., 1966.

\section{UNIVERSITY OF WASHINGTON, Seattle, Washington}

